\title{
THEORETICAL ANALYSIS AND EXPERIMENTAL MEASUREMENT OF DIGITAL MULTI-BEAM PHASED ANTENNA ARRAY IN THE C FREQUENCY RANGE
}

\author{
L. Guoming, P.N. Zakharov, and A.F. Korolev \\ Faculty of Physics, Lomonosov Moscow State University, 119991, GSP-1, 1-2 Leninskiye Gory, Moscow, Russian Federation \\ Email: luguoming.hit@gmail.com
}

Received 5 September 2018; revised 6 November 2018; accepted 2 January 2019

\begin{abstract}
The choice of elements for constructing a phased antenna array providing a relative frequency bandwidth up to $9 \%$ for the transmission or reception of wireless communication system information was made. Superposition of the excitation signals of a flat phased antenna array for a simultaneous emission of several independent beams in a radiation far field was used. Calculation and optimization of the progressive distribution of phase shifts in the excitation signal group in the horizontal and vertical directions of the phased array plane for the radiation (reception) of independent beams in spherical coordinates (azimuth and elevation) in space was performed. An experimental sample of the phased antenna array was developed using microstrip technology, which forms several beams in the radiation far field. An experimental measurement of the voltage standing wave ratio and relative frequency bandwidth of the sample developed by phased array microstrip and printing technology was carried out. The multi-beam phased antenna array will increase the number of subscribers for multi-user systems, as well as increase the energy efficiency for point-to-point communication by transmitting over multiple spatial trajectories.
\end{abstract}

Keywords: phased antenna array, radiation pattern, voltage standing wave ratio

\section{Introduction}

In modern communication systems there is a need to increase throughput [1, 2] The expansion of bandwidth and the increase of transmission power are limited, and in many cases those resources do not allow providing the required throughput [3]. Communication systems with multipleinput multiple-output (MIMO) provide an additional opportunity to increase throughput [2, 何. In most cases MIMO systems use several antennas or multi-element antennas for transmission and reception [2, 4]. Digital multi-beam phased antenna arrays (PAA) are one of the ways to organize the MIMO channel $[5,6$. Multipath propagation of radio waves can be used to improve the energy and spectral transmission efficiency [5]. Such propagation of radio waves is typical for urban environments and radio channels inside buildings; it provides an opportunity to transfer energy along several spatial trajectories. In multiple access systems different directions of transmission and reception can be used to communicate with different subscribers in order to increase the total throughput of the system [7-9]. A multipath radiation pattern for wideband and ultra-wideband PAA can be formed with both analog and digital methods [4, 10, 11]. In analog methods, as a rule, a feeder system, a set of phase shifters and summators on antenna ports are used. The complexity 
of this construction grows significantly with increasing the number of beams and PAA elements, and, as a rule, such schemes are used to form no more than 2-3 rays [4]. The digital circuit of formation of a multipath radiation pattern provides a number of advantages [4, 9, 10, 12, 13]:

- Possibility of a dynamic high-speed change of the beam structure in time in the absence of mechanical rotation, compatibility with modern effective methods of digital modulation; high flexibility in the use of different methods of signal processing without loss in the signal-to-noise ratio; a greater degree of freedom in choosing the shape and direction of beam focusing provided by the program change of phasing weight coefficients;

- Possibility of targeting the partial beams on individual users or their spatially-focused group which ensures the maximum performance of all communication channels;

- Permission to control and compensate any spurious changes in the amplitude and phase of signals along the propagation path between a transmitter and a receiver;

- Possibility of far software reconfiguration of architecture, modernization of signals processing methods and modes of the system operation;

- Today digital beam shaping is the only technology that allows implementing an efficient dynamic adaptation of the service coverage area over ground cells [10].

The microstrip antenna was chosen because of its low thickness, possibility of placing the antenna and path elements on a single printed circuit board and compatibility with modern effective methods of digital modulation [2, 4, 14]. The studies were carried out with the help of simulation in the software package CST Microwave Studio, which uses the numerical solution of Maxwell's equations in an integral form [15].

The structure of this article is as follows: the first part describes the choice of the PAA element, which provided a relative frequency band up to $9 \%$ at $7 \mathrm{GHz}$ central frequency; the second part deals with the comparison of methods for suppressing sidelobes; the third part gives a simulation of a flat microstrip PAA, emitting up to three rays; the fourth part presents the results of experimental measurements of PAA.

\section{Methods}

\subsection{Simulation of the broadband PAA element}

The frequency bandwidth of the microstrip antenna depends on many parameters. These are the geometric shape of the patch element, the dielectric permeability of the substrate, the thickness of the substrate material and the location of the power point. In the research of the frequency bandwidth dependence on the thickness of the substrate a model of a microstrip fan antenna was created. The patch element was in the form of a fan with a radius $r=14 \mathrm{~mm}$, opening angle $\alpha=120^{\circ}$, the thickness of the copper patch and ground 18 microns. The central frequency in the simulation and calculation was $7 \mathrm{GHz}$. The grating size is $14 \times 14 \mathrm{~cm}$; that of the grating patch (element) is $16 \times 12.8 \mathrm{~mm}$, while its thickness is $18 \mu \mathrm{m}$. The feeder line $0.58 \mathrm{~mm}$ wide ensures $50 \Omega$ impedance. The subminiature A-connectors are placed in two sides; the spacing between them is $15 \mathrm{~mm}$ on each side.

In the research of the frequency bandwidth dependence on the dielectric permeability of the substrate material a model of the patch element of a microstrip square antenna was created. The patch element was in the form of a square with the size $20.1 \times 20.1 \mathrm{~mm}$, the thickness of the copper patch and ground 18 microns. The central frequency in the simulation and calculation was $7 \mathrm{GHz}$.

In order to study the frequency bandwidth dependence on the geometric shape of the patch element and the choice of broadband elements, simulation for elements of different shapes was carried out, including square, square with rounded corners, fan, fan with rounded corners, butterfly with rounded corners, circle, hexagon. The following parameters were used in the calculation and simulation: the $7 \mathrm{GHz}$ central frequency, the substrate material Arlon AD255 (with dielectric permeability $\varepsilon=2.55$ and thickness $h=2.032 \mathrm{~mm}$ ), the thickness of the copper patch 18 microns. The simulation results showed that among the studied forms the widest strip was provided by the patch of the element in the form of a square with rounded corners. According to the calculation and the result of simulation, the frequency bandwidth increases with the growth of the dielectric substrate 
thickness and decreases with the growth of dielectric permeability of the substrate material. In view of this, the element in the form of a square with rounded corners was chosen as the main element for constructing arrays, the dielectric material (Arlon AD255, $\varepsilon=2.55, h=2.032 \mathrm{~mm}$ ) was chosen with a low dielectric permeability and a high dielectric substrate thickness. The Arlon AD255 material was also used in the calculation and simulation of the frequency bandwidth depending on a different geometric shape of the patch element. Therefore, the square with rounded corners as the main element and the Arlon AD255 material $(\varepsilon=2.55, h=2.032 \mathrm{~mm})$ as the substrate for the construction of a phased antenna array were chosen. Based on the results of simulation of the radiation pattern and voltage standing wave ratio (VSWR) of the selected form element, the gain was $7.1 \mathrm{~dB}$, the operating frequency band 6.7-7.3 GHz and the central frequency $7 \mathrm{GHz}$.

\subsection{Suppression of the level of PAA sidelobes}

In this work a directional antenna is used and there is a need to reduce the sidelobes (including diffraction) in the space far field [16]. In order to reduce the sidelobes of the radiation pattern in the far field of the antenna array, the following methods are used [16]:

a) reducing distances between the elements to values less than $\lambda / 2$;

b) non-equidistant arrangement of the elements;

c) uneven distribution of currents on the elements;

d) the use of pointed elements.

The methods (a), (b) and (c) were studied in this paper.

\subsubsection{Location of PAA components at distances less than $\lambda / 2$}

At the distance between the elements $d>\lambda / 2$, the radiation far field may contain several radiation maxima equal in intensity to the main maximum [16]. In view of this, one method of reducing sidelobes (including diffraction) is reduction of the distance between the elements less than $\lambda / 2$, that is, $d<\lambda / 2$. Simulation at $d<\lambda / 2$ is carried out to study the level of a side linear PAA with 6 elements emitting in directions of 30,45 and $60^{\circ}$ from the array normal, respectively.
For the horizontal line PAA simulation emitting in directions of 30,45 and $60^{\circ}$ from the normal and distances between the elements of 0.376 , $0.379,0.385,0.397,0.42$ and $0.47 \lambda$, respectively, were chosen. The simulation results showed that at the distance between the elements $d=0.47 \lambda$ in the radiation beam in directions of 30,45 and $60^{\circ}$ from the normal a sidelobe was at the level of -11.5 , -9.9 and $-6.4 \mathrm{~dB}$, respectively. The larger the radiation angle from the normal to the plane of the array, the higher the level of sidelobes is. The lowest level of sidelobes is obtained at the distance between the elements $0.47 \lambda$ for radiation directions of 30 , 45 and $60^{\circ}$ from the normal.

For the simulation of the linear vertical PAA emitting in directions of 30,45 and $60^{\circ}$ from the normal and distances between the elements of $0.376,0.379,0.385,0.397,0.42$ and $0.47 \lambda$, respectively, were chosen. The simulation results showed that at the distance between the elements $d=0.47 \lambda$ in the radiation beam in directions of 30,45 and $60^{\circ}$ from the normal, a sidelobe was at the level of $-12,-10.1$ and $-8.4 \mathrm{~dB}$, respectively. The larger the radiation angle from the normal to the plane of the array, the higher the level of sidelobes is. The lowest level of sidelobes is obtained at the distance of $0.47 \lambda$ between the elements for emission angles of 30,45 and $60^{\circ}$ from the normal.

Based on the results of the linear PAA simulation in the horizontal and vertical directions at the calculated distances between the elements less than $\lambda / 2$, a significant suppression of the sidelobe (including diffraction) in the far radiation field is no more than $-2 \mathrm{~dB}$. At the distance between the elements $0.47 \lambda$ the sidelobe was suppressed as much as possible.

\subsubsection{Non-equidistant arrangement of the elements}

To study possibilities of the method of suppressing sidelobes by positioning the emitters at unequal distances from each other, a model of a linear non-equidistant array with 6 elements was built; the normalized coordinates of the elements were obtained with the calculation: $\xi_{0}=-0.9994$, $\xi_{1}=-0.5343, \xi_{2}=-0.1705, \xi_{3}=0.1713, \xi_{4}=0.5352$ and $\xi_{5}=1$. The distance between the elements is relative to the coordinates $\xi_{0}, \xi_{1}, \xi_{2}, \xi_{3}, \xi_{4}$ and $\xi_{5}$, respectively [16]. In Section 2.1, the distance between the two elements is $0.47 \lambda$, the sidelobe 
level is at least $-2 \mathrm{~dB}$ in the horizontal and vertical directions. In view of this, $0.47 \lambda$ was chosen as the central distance (distance between $\xi_{2}$ and $\xi_{3}$ ) between the elements.

Simulation of the non-equidistant linear PAA with 6 elements in the horizontal direction radiating in directions of 30,45 and $60^{\circ}$ from the PAA normal plane was carried out. The simulation results showed that the level of sidelobes (including diffraction) of the horizontal non-equidistant array with 6 elements did not exceed $-12.8 \mathrm{~dB}$ at radiation in the direction of $30^{\circ}$ from the normal.

Simulation of the non-equidistant linear PAA with 6 elements in the vertical direction radiating in directions of 30,45 and $60^{\circ}$ from the PAA normal plane was carried out. The simulation results showed that the level of sidelobes (including diffraction) of the vertical non-equidistant array with 6 elements did not exceed $-11.1 \mathrm{~dB}$ at radiation in the direction of $30^{\circ}$ from the normal.

According to the results of the linear PAA simulation in the horizontal and vertical directions at unequal distances, a significant suppression of the sidelobe (including diffraction) in the radiation far field is no more than $-12.8 \mathrm{~dB}$, and the number of sidelobes is much larger. The larger the angle of the main lobe radiation from the normal, the larger the sidelobe in the radiation far field is. At unequal distances between the elements in the horizontal direction, the level of the main radiation in the direction of $60^{\circ}$ can already be compared with the level of the sidelobe.

\subsubsection{Uneven distribution of currents on the elements}

To suppress the sidelobes of the antenna array radiation pattern, there is still an important method: uneven distribution of currents on the elements [16, 17]. In this work the Chebyshev distribution was used. In 2.1 and 2.2 it is shown that at equal distances between the elements and the distance between the elements $d$ less than $\lambda / 2$ they do not significantly suppress the sidelobes. At $d=0.47 \lambda$ the maximum suppression of sidelobes is up to $-12 \mathrm{~dB}$. In view of this, in the simulation and calculation the distance between the elements $d=0.47 \lambda$ was chosen to create a linear PAA with the Chebyshev distribution of currents on the elements. According to the Chebyshev function for a linear array with 4 elements and 6 elements, the amplitude distribution of the current on the elements is $1: 3: 3: 1$ and $1: 4: 6: 4: 1$, respectively [16, 17].

In Figs. 1 and 2 the radiation patterns of the $4 \times 1$ array emitting in the direction of $30^{\circ}$ from the normal in the horizontal and vertical directions of the linear array, respectively, are shown.
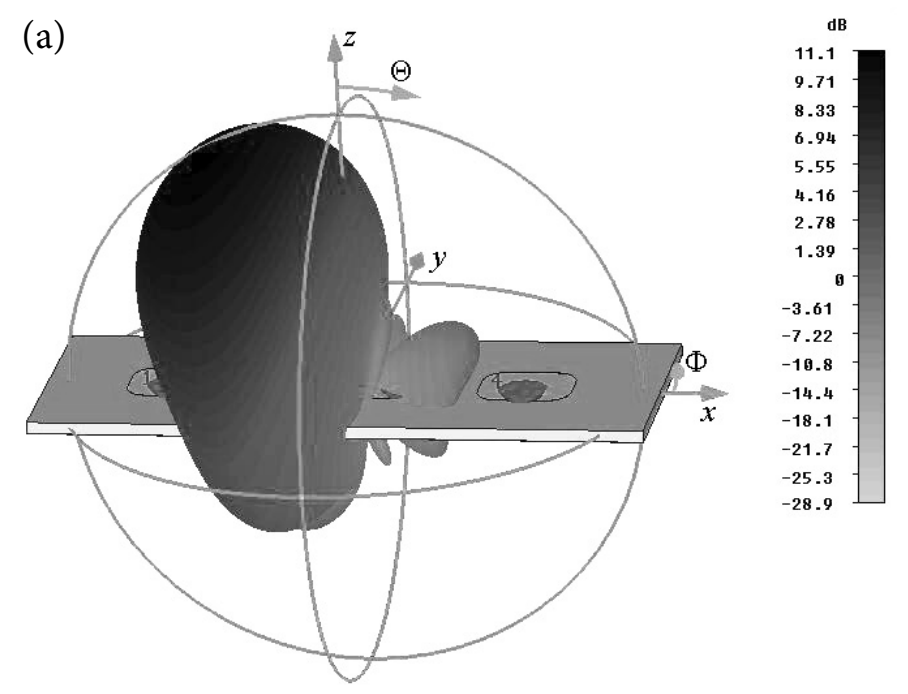

(b)



Theta/Degree vs dB

Fig. 1. Horizontal grating with $4 \times 1$ elements, measurement unit dB: (a) the 3D radiation pattern for far field from a $4 \times 1$ linear horizontal grating (modelling) radiating in the direction $30^{\circ}$; excitation currents through the elements with Chebyshev distribution; (b) the 2D polar antenna pattern; the pattern section in Fig. 1 at Phi $(\Phi)=180^{\circ}$; it is possible to inspect the antenna sidelobe level from this section. 

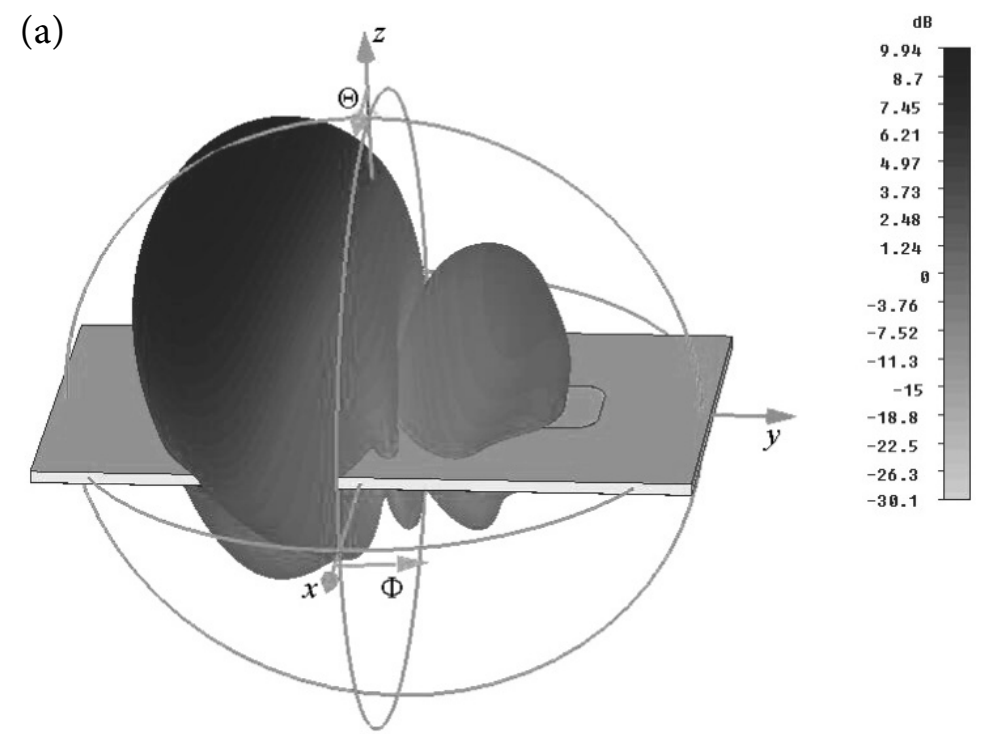

(b)

Far Field Gain ABS $(\mathrm{Phi}=270)$

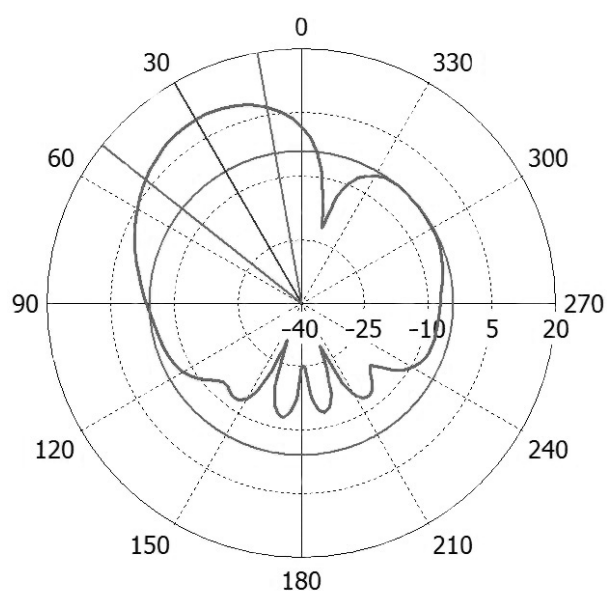

Theta/Degree vs dB

Fig. 2. Vertical grating with $4 \times 1$ elements, measurement unit dB: (a) the 3D radiation pattern for far field from a linear horizontal $4 \times 1$ grating (modelling) radiating in the direction $30^{\circ}$; excitation currents through the elements with Chebyshev distribution; (b) the 2D polar antenna pattern; the pattern section in Fig. 1 at Theta $(\Theta)=180^{\circ}$; it is possible to inspect the antenna sidelobe level from this section.

The results of the calculation and simulation showed that at the radiation of the main beam in the direction of $30^{\circ}$ from the normal horizontal linear array with the Chebyshev distribution of currents on the elements, the sidelobe was suppressed to the level of $-18 \mathrm{~dB}$; the Chebyshev distribution also helped to reduce the level of sidelobes; at the radiation of the main beam in the direction of $30^{\circ}$ from the normal vertical linear array with the Chebyshev distribution of the currents on the elements, the sidelobe was suppressed to the level of $-15 \mathrm{~dB}$.

The results of the calculation and simulation in the horizontal direction showed that the linear array with the Chebyshev distribution of currents on the elements in the direction of $45^{\circ}$ from the normal, the sidelobe was suppressed to the level of $-7 \mathrm{~dB}$, the pattern structure deteriorated, and in the direction of $60^{\circ}$ from the normal, the sidelobe seriously deteriorated. The scanning range of the horizontal linear array is no more than $60^{\circ}$ from the normal in implementation.

The comparison of three methods with the optimized level of sidelobes in directions of 30,45 and $60^{\circ}$ from the normal for the horizontal linear array showed that the Chebyshev distribution of currents on the elements reduced significantly the level of sidelobes in the scan range to $60^{\circ}$ from the normal, and in the range from -30 to $30^{\circ}$ rela- tive to the normal provided the opportunity to radiate energy effectively in the desired direction of space and with the sidelobe level up to $-18 \mathrm{~dB}$.

The results of the calculation and simulation in the vertical direction showed that at the radiation of the main beam of the vertical linear array with the Chebyshev distribution of currents on the elements in the direction of -30 to $30^{\circ}$ relative to the normal, the sidelobe was suppressed to the level of $-15 \mathrm{~dB}$, and in the direction of $45^{\circ}$ from the normal the sidelobe increased up to $-9 \mathrm{~dB}$, it is valid in the implementation; in the direction of $60^{\circ}$ from the normal the sidelobe increased to $-5 \mathrm{~dB}$, the scan range of the vertical linear array is not more than $60^{\circ}$ from the normal, and this is similar to the horizontal array. The comparison of three methods with the optimized level of sidelobes in directions of 30,45 and $60^{\circ}$ for the vertical linear array showed that the Chebyshev distribution of currents on the elements reduced significantly the level of sidelobes in the scan range to $60^{\circ}$ from the normal, and in the range from -30 to $30^{\circ}$ relative to the normal provided the opportunity to radiate energy effectively in the desired direction of space and with the sidelobe level to $-15 \mathrm{~dB}$.

The comparison of three methods with an optimized level of sidelobes in the directions of radiation (reception) relative to the normal 
the horizontal and vertical linear PAA showed that the Chebyshev distribution of currents on the elements reduced significantly the level of sidelobes in the scan range to $60^{\circ}$ from the normal, and in the range from -30 to $30^{\circ}$ relative to the normal provided the opportunity to radiate energy effectively in the desired direction and with a minimum level of sidelobes.

The Chebyshev distribution of currents on the elements allowed reducing significantly the level of sidelobes in the range of emission angles (reception) from -30 to $30^{\circ}$ relative to the normal (up to -18 and $-15 \mathrm{~dB}$ for horizontal and vertical arrays, respectively).

The linear PAA with the Chebyshev distribution of currents on the elements also in the horizontal and vertical directions reduced significantly the sidelobes and provided a range of scanning beams. Therefore, the Chebyshev distribution of currents on the elements and the distance between the elements of $0.47 \lambda$ were chosen for the creation and simulation of the flat PAA.

To reduce the electromagnetic interaction between the antenna elements and the feeder system, a structure consisting of five layers was developed. On the upper layer the antenna patch elements (copper) are placed referred to hereinafter as dielectric-1 (Arlon AD255), ground (copper), dielectric-2 and feeder system. The location of the antenna elements and the feeder system on separate layers allows reducing the electromagnetic interaction between them [17].

\section{Results and discussion}

\subsection{Simulation of the flat microstrip PAA emitting up to three rays}

Key parameters for creating a flat PAA were selected and optimized. The array elements in the form of a square with rounded corners were created on the substrate Arlon AD255 $\left(\varepsilon_{\mathrm{r}}=2.55\right)$ with a thickness of $2.032 \mathrm{~mm}$; the distance between the elements of $0.47 \lambda$ in the horizontal and vertical directions to the plane of PAA and the Chebyshev distribution of currents on the elements in the horizontal and vertical directions of PAA were used. These selected key parameters for creating a flat PAA emitting three rays were used for simulation.

\subsubsection{Simulation of the $4 \times 1$ multipath linear array}

The number of emitted rays depends on the number of excitation signals. Superposition of excitation signals was used to emit several independent beams simultaneously. The superposition of two groups of excitation signals was used to form two beams in the simulation of the linear horizontal array. The first group of excitation signals with a phase shift $\Delta \alpha$ formed the first beam; the second group of excitation signals with an additional phase shift $\Delta \beta$ formed the second beam. The amplitude weighting coefficients after the superposition of two sets of excitation signals corresponded to the Chebyshev distribution 1:3:3:1 [16, 17]. The results of the calculation and simulation showed that the radiation patterns in the $3 \mathrm{D}$ representation of horizontal and vertical linear arrays $4 \times 1$ with the Chebyshev distribution of currents on the elements emitting two beams in the predetermined directions are relative to the normal.

\subsubsection{Simulation of the $4 \times 4$ multipath flat $P A A$}

A flat array with $4 \times 4$ dimension was developed. The direction of radiation (reception) in azimuth and in elevation depends on the progressive distribution of phase displacements in the group of excitation signals in the horizontal (on the $x$ axis) and vertical (on the $y$ axis) directions of the PAA plane [17]; the weighting coefficients in the superposition of excitation signals corresponded to the Chebyshev distribution in the horizontal and vertical directions to reduce the level of sidelobes.

In the simulation and calculation a group of 16 excitation signals for forming a single beam was used; the phase shift in the horizontal direction (on the $x$ axis) was $\Delta \varphi 1$ for the formation of maximum radiation in the direction of azimuth $=15^{\circ}$; the phase shift in the vertical direction (on the $y$ axis) was $\Delta \varphi 2$ for the formation of maximum radiation in the direction of the elevation angle $=-30^{\circ}$; the weighting amplitude coefficients in the horizontal and vertical directions corresponded to the Chebyshev distribution 1:3:3:1. Shown in Fig. 3(a) is a $3 \mathrm{D}$ radiation pattern in the orientation of a linear scale of the flat array emitting one beam in the direction of the elevation angle $=-30^{\circ}$, azimuth $=15^{\circ}$. 

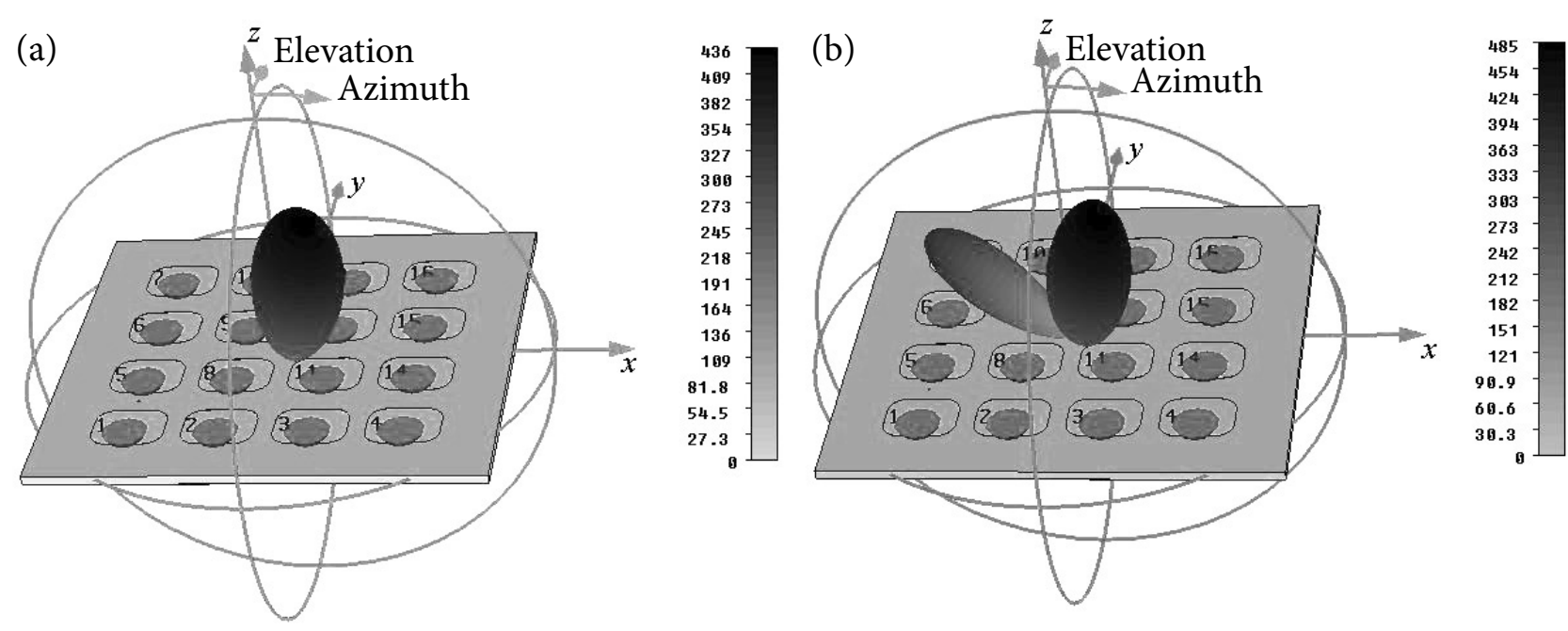

Fig. 3. 3D directional pattern of a flat $4 \times 4$ grating at the linear scale radiating one beam (a) and two beams (b) in the specified directions; excitation currents through the elements with the Chebyshev coefficients 1:3:3:1 in the horizontal (on the $x$ axis) and vertical (on the $y$ axis) directions.

The superposition of two groups of excitation signals was used to form two beams simultaneously: the first group of 16 excitation signals to form a beam in the direction of the elevation angle $=0^{\circ}$, azimuth $=-45^{\circ}$ and the second group of 16 excitation signals to form a beam in the direction of the elevation angle $=-30^{\circ}$, azimuth $=15^{\circ}$. The phase shift in the first group on the $x$ axis was $\Delta \varphi 1$ for the formation of maximum radiation in the direction of azimuth $=15^{\circ}$; the phase shift in the direction of the $y$ axis was missed $\left(\Delta \varphi 2=0^{\circ}\right)$ for the formation of maximum radiation in the direction of the elevation angle $=0^{\circ}$. For the formation of the second beam in the direction of the elevation angle $=-30^{\circ}$, azimuth $=15^{\circ}$ the phase shift in the second group of 16 excitation signals was $\Delta \varphi 3$ on the $x$ axis to form the maximum radiation in the direction of azimuth $=15^{\circ}$ and $\Delta \varphi 4$ on the $y$ axis to form the maximum radiation in the direction of the elevation angle $=-30^{\circ}$. After the superposition of excitation signal groups the signals were weighed with the coefficients 1:3:3:1 in the horizontal (on the $x$ axis) and vertical (on the $y$ axis) directions. Shown in Fig. 3(b) are a $3 \mathrm{D}$ radiation pattern in a linear scale of the flat array emitting simultaneously two independent beams in the directions of the elevation angle $=0^{\circ}$, azimuth $=-45^{\circ}$ and the elevation angle $=-30^{\circ}$, azimuth $=15^{\circ}$.

For formation of simultaneously independent three beams the superposition of three groups of excitation signals was used, each of them was responsible for formation of a certain beam and consisted of 16 excitation signals. After superposition of excitation signal groups, the signals were weighed with the Chebyshev coefficients 1:3:3:1 in the horizontal (on the $x$ axis) and vertical (on the $y$ axis) directions.

The direction of radiation (reception) in azimuth and elevation depends on the progressive distribution of phase shifts in the excitation signal group in the horizontal (on the $x$ axis) and vertical (on the $y$ axis) directions [17]; the weighting coefficients in the superposition of excitation signals corresponded to the Chebyshev distribution in the horizontal and vertical directions to reduce the level of sidelobes [16, 17]. To form beams, the superposition of groups of excitation signals was used, each of them was responsible for formation of a certain beam and consisted of 16 excitation signals. The $3 \mathrm{D}$ radiation patterns on a linear scale of the flat PAA emitting independent beams in the predetermined directions are shown in Figs. $3(a, b)$, respectively.

The Chebyshev distribution was used in the horizontal and vertical directions of the grating plane; the pattern is represented in the spherical coordinate system; the angle of elevation and azimuth show the direction of radiation beams; the values in the linear pattern are normalized power; there is no measurement unit. 
3.2. Experimental measurement of VSWR and the frequency bandwidth of the developed PAA

In order to reduce the electromagnetic interaction between the emitting elements of PAA and feeders, a structure consisting of seven layers was developed. On the top layer there are the antenna elements, referred to hereinafter as dielectric-1 (Arlon AD255 with dielectric permeability $\varepsilon=2.55$ and thickness of $2.032 \mathrm{~mm}$ ), ground (copper), a dielectric binder-2 (FR-4 with dielectric permeability $\varepsilon=4.34$ and thickness of $0.1 \mathrm{~mm}$ ), ground (copper), dielectric-3 (Rogers 4003 with dielectric permeability $\varepsilon=3.55$ and thickness of $0.305 \mathrm{~mm}$ ), feeder lines (copper). The location of the antenna elements and the feeder system on separate layers allows reducing the electromagnetic interaction between them [11, 17-19].

Shown in Fig. 4 are the results of the experimental measurements of reflection coefficients on ports and bandwidth of the developed PAA with the help of a vector analyzer of circuits Rohde of Schwarz ZVA40. When measuring the first grating element, the other grating elements are free; when measuring the second grating element, the other grating elements are free; ... ...; when measuring the sixteenth grating element, the other grating elements are free. The cable is connected from the Rohde \& Schwarz ZVA40 output to the grating element (Fig. 5). The results of

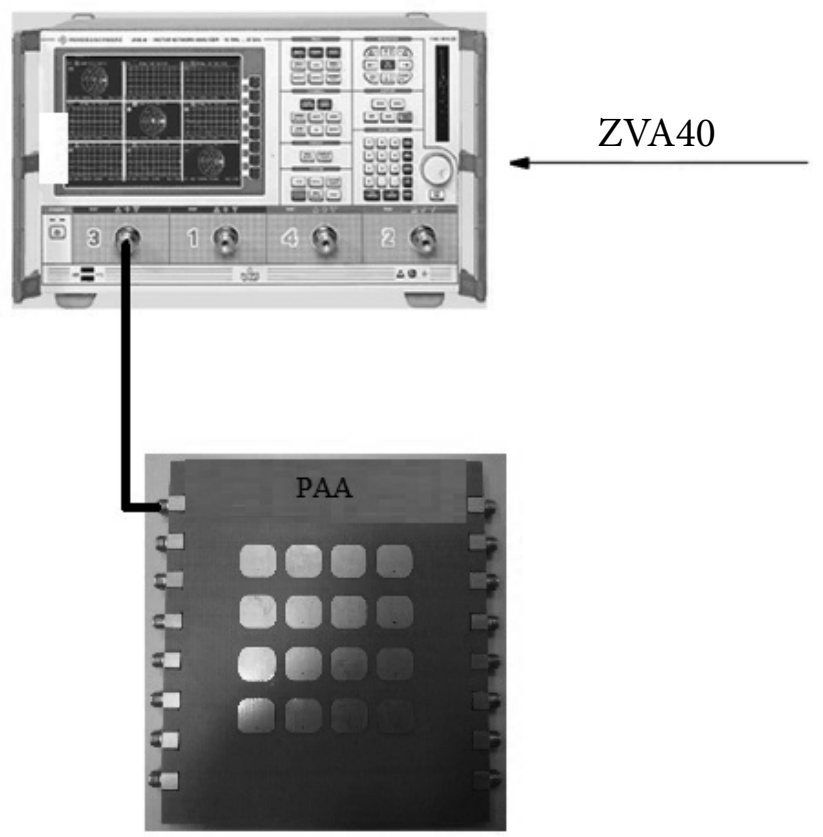

Fig. 5. The measurement circuit of VSWR and the frequency band of the developed PAA using a vector network analyzer Rohde \& Schwarz ZVA40.

the measurement showed that the PAA element operated at $7.1 \mathrm{GHz}$ central frequency, slightly higher than the values in the simulation, the relative frequency band at the level of $-10 \mathrm{~dB}$ was up to $8.5 \%$. Each PAA element provided a satisfactory gain value up to $8 \mathrm{~dB}$ in the radiation far field.

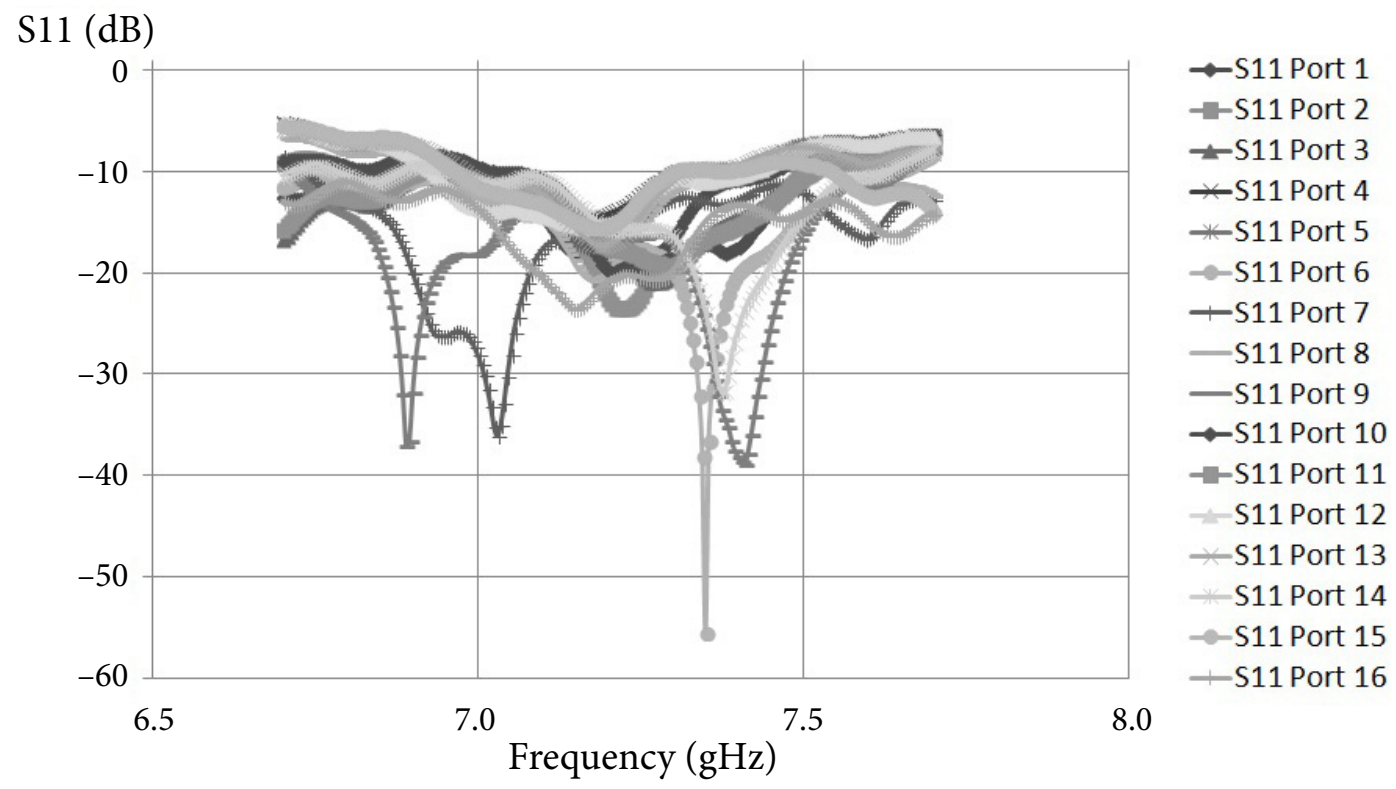

Fig. 4. The experimental measurements of VSWR for each element of the developed test grating. For each element of the made grating, the band centre, frequency bandwidth and resonance depth (in $\mathrm{dB}$ ) are examined from VSWR. 
The Arlon AD255 material (dielectric permeability $\varepsilon=2.55$ and thickness of 2.032) and the patch element in the form of a square with rounded corners were chosen to construct the PAA element; the optimized element provides the highest relative frequency bandwidth about $9 \%$ at the $7 \mathrm{GHz}$ central frequency at the VSWR 2.0 level; the maximum reduction in the level of the sidelobes of the radiation pattern in the flat PAA up to $-18 \mathrm{~dB}$ at the $7 \mathrm{GHz}$ operating frequency; the flat PAA of $4 \times 4$ dimension was developed, which ensured the emission of several independent beams simultaneously in the specified directions of the space far field; the experimentally measured PAA frequency bandwidth was slightly less, and the central frequency was $\sim 2 \%$ higher than the values obtained in the simulation, which can be explained by errors in the manufacture of the prototype.

\section{Conclusions}

The element for the construction of antenna arrays, providing a wide relative frequency bandwidth, was selected, which allows increasing the bandwidth of wireless channels. For a digital multi-beam PAA it is advisable to use the Chebyshev distribution of currents on the elements to suppress the sidelobes, which allows obtaining a greater number of rays. In practice, the multibeam PAA will increase the number of subscribers for multi-user systems, as well as increase the energy efficiency of point-to-point communication through the transmission of multiple spatial trajectories. A digital scheme of the formation of a multi-beam radiation pattern provides a dynamic high-speed change of the beam structure in time in the absence of mechanical rotation.

The relative frequency bandwidth increased with growth in the thickness of the dielectric substrate and decreased with growth in values of the dielectric permeability, the greatest strip provides an element in the form of a square with rounded corners; the Chebyshev distribution of currents on the elements when the distance between the PAA elements was $0.47 \lambda$ maximally suppressed the sidelobe level and diffraction up to $-18 \mathrm{~dB}$; the number of independent PAA beams depends on the amount of superposition of groups of excitation signals to the input ports of PAA; the phase shift distribution of each group of excitation signals in the horizontal and vertical directions in the PAA plane (on the axes OX and OY) determined the direction of the beam radiation; the frequency bandwidth of the developed PAA provided the transmission (reception) of energy for the communication system.

\section{References}

[1] M.A. Jensen and J.W. Wallace, A review of antennas and propagation for MIMO wireless communications, IEEE Trans. Antennas Propag. 52, 2810-2812 (2004).

[2] J.B. Andersen, Array gain and capacity for known random channels with multiple element arrays at both ends, IEEE J. Select Areas Commun. 18, 2172-2178 (2000).

[3] J.W. Wallace, M.A. Jensen, A.L. Swindlehurst, and B.D. Jeffs, Experimental characterization of the MIMO wireless channel: Data acquisition and analysis, IEEE Trans. Wireless Commun. 2, 335-343 (2003).

[4] A.F. Molisch and M.Z. Win, MIMO systems with antenna selection, IEEE Microw. Mag. 5(1), 4748 (2004).

[5] L. Zheng and D. Tse, Diversity and multiplexing: A fundamental tradeoff in multiple antenna channels, IEEE Trans. Inf. Theory 49, 1073-1096 (2003).

[6] M. Lienard, P. Degauque, J. Baudet, and D. Degardin, Investigation on MIMO channels in subway tunnels, IEEE J. Select Areas Commun. 21, 332-339 (2003).

[7] J. Zhang, Z. Zheng, Y. Zhang, J. Xi, X. Zhao, and G. Gui, 3D MIMO for 5G NR: Several observations from 32 to massive 256 antennas based on channel measurement, IEEE Commun. Mag. 6(3), 62-70 (2018).

[8] M.M. Lodro, N. Majeed, A.A. Khuwaja, A.H. Sodhro, and S. Greedy, in: Proceedings of the 2018 International Conference on Computing, Mathematics and Engineering Technologies (iCoMET) (2018) pp. 1-5.

[9] S.F. Jilani and A. Alomainy, Millimetre-wave T-shaped MIMO antenna with defected ground structures for $5 \mathrm{G}$ cellular networks, IET Microw. Antenna P. 12(5), 672-677 (2018). 
[10]B.M. Petrov, Electrodynamics and Propagation of Radio Waves (Hotline-Telecom, Moscow, 2007) [in Russian].

[11]L. Guoming, P.N. Zakharov, and A.P. Sukhorukov, Simulation of low-sidelobe phased antenna array with circular polarization, Moscow Univer. Phys. Bull. 68(6), 437-442 (2013).

[12]V.I. Slyusar, Digital antenna arrays are the future of radiolocation, Electronics: NTB 3, 42-47 (2001) [in Russian].

[13]V.I. Slyusar, Phased antenna array of Thuraya system, Netw. Telecommun. 5, 54-58 (2002) [in Russian].

[14]K.A. Geniatulin and V.I. Nosov, Planning of satellite communication systems with zonal service, Vestnik SibSUTIS 4, 11-22 (2009) [in Russian].

[15]P.N. Zakharov, R.A. Dudov, E.V. Mikhailov, A.F. Korolev, and A.P. Sukhorukov, in: Proceedings of the 2009 Loughborough Antennas \& Propagation Conference (LAPC) (Loughborough, UK, 2009) pp. 369-372.

[16]O.G. Vendik and M.D. Parnes, Antennae with Electric Scanning (Radio and Communication, Moscow, 2001) [in Russian].

[17]A.A. Skvortsov, D.E. Pshonkin, M.N. Lukyanov, and M.R. Rybakova, Deformations of aluminum alloys under the influence of an additional load, Periódico Tchê Química 15(30), 421-427 (2018).

[18]A.J. Fenn, D.H. Temme, W.P. Delaney, and W.E. Courtney, The development of phased-array radar technology, Lincoln Lab. J. 12, 320-340 (2000).

[19]A.S. Daryoush, in: Proceedings of the 2008 IEEE Radio and Wireless Symposium (2008) pp. 831834.

\title{
SKAITMENINIŲ DAUGIAPLUOŠČIŲ FAZUOTŲ ANTENŲ RINKINIO TEORINE் ANALIZEE IR EKSPERIMENTINIS MATAVIMAS C DAŽNIŲ RUOŽE
}

\author{
L. Guoming, P.N. Zakharov, A.F. Korolev
}

Maskvos valstybinio Lomonosovo universiteto Fizikos fakultetas, Maskva, Rusijos Federacija 